\title{
HOXC6 promotes gastric cancer cell invasion by upregulating the expression of MMP9
}

\author{
SHI-WEI CHEN ${ }^{1 *}$, QING ZHANG $^{1 *}$, ZHI-FENG XU $^{1}$, HAI-PING WANG $^{1}$, \\ $\mathrm{YI} \mathrm{SHI}^{1}$, FENG XU ${ }^{1}$, WEN-JIAN ZHANG ${ }^{2}$, PING WANG $^{3}$ and YONG LI ${ }^{1}$ \\ ${ }^{1}$ Department of Medical Oncology, Gongli Hospital, Shanghai 200135; \\ ${ }^{2}$ Department of Clinical Medicine, School of Medicine, Shandong University, Jinan, Shandong 250012; \\ ${ }^{3}$ Department of Pathology, Shanghai Ninth People's Hospital Affiliated Shanghai Jiaotong University School of Medicine, \\ Shanghai 200011, P.R. China
}

Received June 17, 2015; Accepted July 4, 2016

DOI: $10.3892 / \mathrm{mmr} .2016 .5640$

\begin{abstract}
Previous studies have demonstrated that the homoebox C6 (HOXC6) gene is highly expressed in gastric cancer tissues and is associated with the depth of tumor invasion, and is associated with poor prognosis of gastric cancer patients expressing HOXC6. The present study investigated the effect and underlying mechanism of HOXC6 on the proliferation and metastasis of gastric cancer cells in vitro. Reverse transcription-quantitative polymerase chain (PCR) reaction was used to investigate the expression levels of HOXC6 in different gastric cancer cell lines and the effect of different levels of expression on the proliferation of gastric cancer cells was determined by cell growth curve and plate colony formation. The effect of HOXC6 on the anchorage-independent proliferation of gastric cancer cells was determined by soft agar colony formation assay while the Transwell invasion assay was used to investigate the effect of different levels of HOXC6 expression on the invasive and metastatic abilities of gastric cancer cells. Semi-quantitative PCR was used to detect the effect of different levels of HOXC6 expression on the expression of matrix metalloproteinase (MMP)2 and MMP9 in gastric cancer cells. Immunoblotting was used to assess MMP9 signaling in the gastric cancer cells. The HOXC6 gene is highly expressed in the majority of the gastric cancer cell lines. Overexpression of HOXC6 promoted gastric cancer
\end{abstract}

Correspondence to: Dr Yong Li, Department of Medical Oncology, Gongli Hospital, 219 Miaopu Road, Shanghai 200135, P.R. China

E-mail: liyjdjy@gmail.com

Dr Ping Wang, Department of Pathology, Shanghai Ninth People's Hospital Affiliated Shanghai Jiaotong University School of Medicine, 639 Zhizaoju Road, Shanghai 200011, P.R. China

E-mail:wangpsdu@gmail.com

*Contributed equally

Key words: HOXC6, gastric cancer, invasion, MMP9 cell proliferation and colony formation ability while HOXC6 downregulation inhibited cell proliferation and clone forming ability. HOXC6 overexpression also enhanced the soft agar colony formation ability of gastric cancer cells while HOXC6 downregulation decreased the colony formation ability. Upregulated HOXC6 increased the migration and invasion abilities of gastric cancer cells while interfering with HOXC6 expression inhibited the migration and invasion of the gastric cancer cells. The expression of MMP9 was enhanced with an upregulation of HOXC6 expression while HOXC6 downregulation lowered MMP9 gene expression levels. Increased expression of HOXC6 in gastric cancer cell lines significantly activated extracellular signal-regulated kinase signaling and upregulated MMP9. The HOXC6 gene promotes the proliferation of gastric cancer cells while upregulation of MMP9 promotes migration and invasion of gastric cancer cells.

\section{Introduction}

Gastric cancer is currently one of the malignant tumors with highest morbidity and mortality rate worldwide and is one of the most common malignant tumors in East Asia $(1,2)$. As a result of the challenges in the early diagnosis of gastric cancer, the majority of patients are diagnosed at an advanced stage, reducing the opportunity for prompt diagnosis and treatment (3). Advanced stage gastric cancer has marked invasive ability and poor prognosis, with a five-year survival rate of only $25-35 \%$ (4).

The homeobox (HOX) gene family is an important factor in the regulation of embryonic development in which the gene family comprises a group of transcription factors that regulate the transcription of the downstream target genes by specific DNA binding $(5,6)$. Previous studies have demonstrated that the HOX family gene is abnormally expressed in a variety of tumors, including colorectal, breast, lung, and ovarian tumors (7-10). It was observed that the HOXC6 gene expression in esophageal cancer tissue was significantly higher than in the tumor adjacent tissues (11). Our previous study also indicated that the level of expression of the HOXC6 gene in gastric cancer tissue is significantly elevated and associated with the poor prognosis of the patients (12). Thus, the present study 
hypothesizes that the HOXC6 gene may be the key regulatory gene for digestive tract tumors (13).

The current study expands on our previous study concerning the association between HOXC6 gene expression and depth of tumor invasion (12), it aims to further elucidate the association and possible underlying mechanisms between HOXC6 gene expression and gastric cancer cell invasion and metastasis.

\section{Materials and methods}

Gastric cancer cell lines. The present study used four different gastric cancer cell lines with different degrees of differentiation, including the MGC-803 well-differentiated gastric adenocarcinoma cell line, the SGC-7901 moderately differentiated gastric adenocarcinoma cell line, the BGC-823 poorly differentiated gastric adenocarcinoma cell line, the HGC-27 undifferentiated gastric adenocarcinoma cell line and the GES-1 normal gastric epithelial cell line (Stem Cell Bank, Chinese Academy of Sciences, Shanghai, China). Cell culture was performed using Dulbecco's modified Eagle's medium (Hyclone; GE Healthcare Life Sciences, Logan, UT, USA) containing $10 \%$ fetal bovine serum (Gibco; Thermo Fisher Scientific, Inc., Waltham, MA, USA) and the cells were incubated at $37^{\circ} \mathrm{C}$ and $5 \% \mathrm{CO}_{2}$.

Antibodies. Anti-HOXC6 mouse monoclonal antibody (sc-376330; $1: 500)$ and anti- $\beta$-actin mouse monoclonal antibody (sc-47778; 1:1,000) were purchased from Santa Cruz Biotechnology, Co., Ltd. (Dallas, TX, USA). Mouse monoclonal anti-Flag antibody (F1804; 1:1,000) was purchased from Sigma-Aldrich (St. Louis, MO, USA). Rabbit polyclonal phosphorylated-extracellular signal-regulated kinase (ERK; \#9101; 1:1,000) (Thr202/Tyr204) and rabbit monoclonal MMP9 (\#13667; 1:1,000) antibodies were purchased from Cell Signaling Technology, Inc. (Danvers, MA, USA).

RNA extraction. Total RNA extraction was performed using the TRIzol reagent (Invitrogen; Thermo Fisher Scientific, Inc.) according to the manufacturer's protocol. The concentration and quality of the extracted RNA was determined and monitored by using the NanoDrop 1000 spectrophotometer (Thermo Fisher Scientific, Inc., Wilmington, DE, USA).

Reverse transcription-polymerase chain reaction (RT-PCR) and quantitative PCR ( $q P C R)$. M-MLV reverse transcriptase (Promega Corporation, Madison, WI, USA) was used in a $20 \mu \mathrm{l}$ reaction volume, with $2 \mu \mathrm{g}$ of total RNA added to the reaction template. The reverse transcription reaction was conducted at $37^{\circ} \mathrm{C}$ for $60 \mathrm{~min}$ and $70^{\circ} \mathrm{C}$ for $5 \mathrm{~min}$. Thermal Cycler Dice ${ }^{\mathrm{TM}}$ Detection system and SYBR Premix Ex Taq $^{\text {TM }}$ II dye (Takara Bio, Inc., Otsu, Japan) were used to perform the RT-PCR and qPCR. The reaction conditions were as follows: For RT-PCR, initial denaturation at $94^{\circ} \mathrm{C}$ for $5 \mathrm{~min}, 25-35$ amplification cycles of $94^{\circ} \mathrm{C}$ denaturation for $30 \mathrm{sec}, 55^{\circ} \mathrm{C}$ annealing for $30 \mathrm{sec}$ and $72^{\circ} \mathrm{C}$ extension for $30 \mathrm{sec}$, final extension was conducted at $72^{\circ} \mathrm{C}$ for $10 \mathrm{~min}$; for qPCR, initial denaturation at $95^{\circ} \mathrm{C}$ for $30 \mathrm{sec}$, then 40 amplification cycles of $95^{\circ} \mathrm{C}$ denaturation for $5 \mathrm{sec}, 60^{\circ} \mathrm{C}$ annealing and extension for $30 \mathrm{sec}$. The primers used were as follows: HOXC6 amplification: Forward, 5'-ACAGACCTC AATCGCTCAGG-3' and reverse, 5'-GTACCGCGAGTA GATCTGGC-3' for HOXC6; forward, 5'-GGATGGCAA GTACGGCTTCT-3' and reverse, 5'-GTTCCCACCAAC AGTGGACA-3' for MMP2; forward, 5'-AAGGATGGGAAG TACTGGCG-3' and reverse, 5'-ACCTGGTTCAACTCA CTCCG-3' for MMP9; and forward, 5'-AGAGCCTCGCCT TTGCCGATCC-3' and reverse, 5'-CTGGGCCTCGTCGCC CACATA-3' for $\beta$-actin. The bands observed were quantified using optical density scanning.

Recombinant plasmid construction. Full-length HOXC6 open reading frame (708 bp; GenBank accession number, NM_004503) was obtained from the RNA amplification of the SGC-7901 gastric cancer cell line and the primers used were: Forward, 5'-GCCAAGCTTATGAATTCCTACTTC ACTAACCCTTCCT-3' and reverse, 5'-TAAGGATCCTCA CTCTTTCTGCTTCTCCTCTTCTG-3'. The PCR product was then cloned into the pFLAG-CMV2 Expression Vector (Sigma-Aldrich) using the by the restriction enzymes KpnI and BamHI from the DNA Ligation kit (Takara Biotechnology Co., Ltd., Dalian, China).

RNA interference (RNAi). The downregulation of endogenous HOXC6 was attained by the insertion of DNA fragments (obtained using the DNA Ligation kit) of synthetic coding short hairpin RNA (shRNA) into a $\mathrm{pENTR}^{\mathrm{TM}} / \mathrm{H} 1 / \mathrm{TO}$ plasmid (Thermo Fisher Scientific, Inc.). The sequences were as follows: Forward, 5'-CACCGCCTCAATTCCACCGCC TATTTCGAAAAATAGGCGGTGGAATTGAGG-3' and reverse, 5'-AAAACCTCAATTCCACCGCCTATTTTTCGA AATAGGCGGTGGAATTGAGGC-3' for shRNA-1; and forward, 5'-CACCGCACCGTCAGTGTTCCTATTTCGAAA AATAGGAACACTGACGGTGC-3' and reverse, 5'-AAA AGCACCGTCAGTGTTCCTATTTTTCGAAATAGGAAC ACTGACGGTGC-3' for shRNA-2. The internal reference was an unrelated shRNA-negative control (NC) sequence.

Cell transfection. Lipofectamine ${ }^{\mathrm{TM}} 2000$ transfection reagent (Invitrogen; Thermo Fisher Scientific, Inc.) was used to perform transfection of the cells $\left(\sim 5 \times 10^{5}\right)$ with the recombinant and shRNA plasmids, and the procedure was performed according to the manufacturer's protocol.

Cell proliferation. The transient transfected cells were cultured in 96-well plates for 6 days and Cell Counting Kit-8 (Dojindo Molecular Technologies, Inc., Kumamoto, Japan) was used according to the manufacturer's protocol to determine cell activity. Optical density was measured at a wavelength of $450 \mathrm{~nm}$ on an microplate reader.

Colony formation experiments. The gastric cancer cells transfected with overexpressed HOXC6 plasmid (with empty vector as the control) or HOXC6 shRNA (with shRNA-NC as the control) were inoculated in $100 \mathrm{~mm}$ culture dishes and clone culture was conducted using Zeocin (Thermo Fisher Scientific, Inc.) added to the culture medium for stress screening. After 3-4 weeks at $37^{\circ} \mathrm{C}$ and $5 \% \mathrm{CO}_{2}$, crystal violet staining solution was used to stain and count the proliferated colonies using a light micrcoscope. 
The soft agar colony formation assay consisted of inoculating and culturing the transfected cells into 24 well-plates containing $0.5 \%$ top agar and $1 \%$ base agar. The cell culture was performed for $\sim 3-4$ weeks at $37^{\circ} \mathrm{C}$ and $5 \% \mathrm{CO}_{2}$ and the colony count was performed using a light microscope.

Immunoblotting analysis. Total protein extracts were collected with a $2 \mathrm{X}$ lysis buffer [50 mM Tris- $\mathrm{HCl}$ (pH 6.8), 2\% SDS, $10 \%$ 2-mercaptoethanol, $10 \%$ glycerol, and protease inhibitor cocktail; Sigma-Aldrich). The protein samples (30 $\mu \mathrm{g})$ were subjected to $8 \%$ SDS-PAGE, and the nitrocellulose membranes were blocked with phosphate-buffered saline (PBS) with $5 \%$ non-fat milk, incubated with the primary antibodies at $4^{\circ} \mathrm{C}$ overnight, followed by reaction with the following fluorescent secondary antibodies: Donkey anti-mouse (610-745-124) and donkey anti-rabbit (611-744-127) IgG (H\&L) DyLight ${ }^{\mathrm{TM}}$ 680-conjugated pre-adsorbed antibodies (1:10,000; Rockland Immunochemicals, Inc., Pottstown, PA, USA). PBS containing $0.1 \%$ Triton X-100 was used for washing, and $\beta$-actin served as an internal control. The protein samples were quantified using the Bicinchoninic Acid Protein Assay kit (Thermo Fisher Scientific, Inc.).

Transwell assay. Cells were seeded onto the upper chamber of Transwell membrane (migration assay; Corning Incorporated, Cambridge, MA, USA) or Matrigel-coated membrane (invasion assay; $5 \mathrm{mg} / \mathrm{ml}$ ) in serum-free medium, medium containing $10 \%$ fetal bovine serum was added to the lower chamber as a chemoattractant. Subsequent to culture for $48 \mathrm{~h}$, migratory cells were fixed with methanol, stained with crystal violet and counted. The number of cells that had moved across the microporous membrane were quantified, with the mean number of cells counted in each of 5 random fields. Images were captured with a microscope at a magnification of $\mathrm{x} 400$ (Nikon Corporation, Tokyo, Japan). Each experiment was repeated 3 times.

Statistical analysis. The results were expressed as the mean \pm standard deviation. The two sets of data were analyzed using t-test with paired data analyzed using a paired t-test. Multiple groups of data were analyzed using one-way analysis of variance. GraphPad Prism software, version 5.0 (GraphPad Software, Inc., La Jolla, CA, USA) was used for statistical analysis. $\mathrm{P}<0.05$ was considered to indicate a statistically significant difference.

\section{Results}

Upregulation of HOXC6 expression was observed in the gastric cancer cell lines. The results of a previous study demonstrated that the expression of HOXC6 gene is upregulated in gastric cancer clinical samples (12). The present study selected four gastric cancer cell lines with different degrees of differentiation, the MGC-803 well-differentiated gastric carcinoma cell line, the moderately differentiated SGC-7901 gastric adenocarcinoma cell line, the BGC-823 poorly differentiated gastric adenocarcinoma cell line and the HGC-27 undifferentiated gastric adenocarcinoma cell line. The level of HOXC6 expression in the cell lines was determined by RT-PCR and qPCR and compared with the HOXC6 expression level in the
GES-1 normal gastric epithelial cell line. It was observed that compared with the GES-1 cells, the HOXC6 mRNA expression level was significantly elevated in the different gastric cancer cell lines (Fig. 1A and B).

Overexpression of HOXC6 in vitro promoted cell proliferation and colony formation in gastric cancer. In order to investigate the role of HOXC6 in gastric cancer cells, the recombinant plasmid CMV2-HOXC6 was transiently transfected in the GES-1 cell line and the four human gastric cancer cell lines (Fig. 2). The effect of the CMV2-HOXC6 plasmid on expression was evaluated (Figs. 2A and 3A), and it was observed that compared with the plasmid transfected with the empty vector, HOXC6 overexpression significantly promoted cell growth in GES- 1 cells at days 4 and $6(\mathrm{P}<0.05$ and $\mathrm{P}<0.01$, respectively; Fig. 2B), and cell growth and colony formation in the four gastric cancer cells $(\mathrm{P}<0.05$ and $\mathrm{P}<0.01$; Fig. $3 \mathrm{~B}$ and $\mathrm{C})$. In the soft agar colony formation assay, it was demonstrated that compared with the cells transfected with the empty vector, the four gastric cancer cell lines exogenously expressing HOXC6 exhibited marked invasive grow th with a significantly increased number of colonies $(\mathrm{P}<0.05$; Fig. 3D). This type of marked anchorage-dependent and -independent growth ability suggests that HOXC6 is exerts an important effect in maintaining cell proliferation.

Downregulation of HOXC6 inhibited cell proliferation and colony formation in gastric cancer in vitro. In order to further investigate the role of HOXC6 in cell proliferation and colony formation in gastric cancer, shRNA constructed from $\mathrm{pENTR}^{\mathrm{TM}} / \mathrm{H} 1 / \mathrm{TO}$ plasmid was used to downregulate the expression of endogenous HOXC6 in the cell lines. As expected, compared with the cells transfected with an unrelated shRNA-NC sequence, the two shRNA molecules were able to significantly downregulate the expression of endogenous HOXC6 and inhibited the growth of cells $(\mathrm{P}<0.05$ and $\mathrm{P}<0.01$; Figs. 2, 4A and B). In order to determine the role of HOXC6 in clone formation, the shRNA plasmids were transfected into the four gastric cancer cell lines. The results demonstrated that, compared with the control group transfected with shRNA-NC, the two shRNA molecules significantly inhibited clone forming ability of the cells $(\mathrm{P}<0.05$ and $\mathrm{P}<0.01$; Fig. 4C). Furthermore, compared with cells transfected with negative control shRNA-NC, the downregulation of HOXC6 expression decreased the anchorage-independent growth ability of the gastric cancer cells in soft agar $(\mathrm{P}<0.05$ and $\mathrm{P}<0.01$; Fig. 4D). The above data suggest that endogenous HOXC6 is important in the proliferation and colony formation of gastric cancer cells.

Expression of HOXC6 promoted migration and invasion of gastric cancer cells. Previous research has demonstrated that the expression of the HOXC6 gene in gastric cancer patients was associated with the depth of tumor invasion (12), indicating that HOXC6 may be associated with tumor cell migration and invasion. The results of the previously described soft agar colony formation assay also suggest that HOXC6 enhances the anchorage-independent growth of gastric cancer cells (Figs. 3D and 4D), further implying that cells with high HOXC6 expression levels were prone to metastasis. In order to investigate 
A

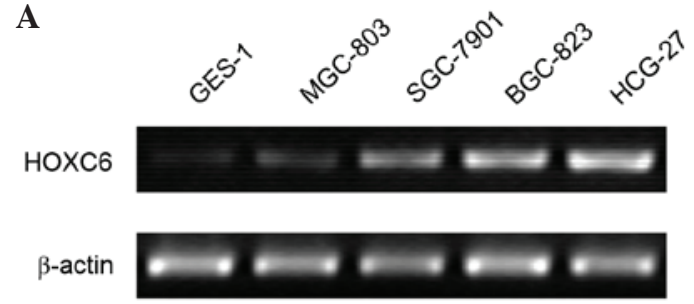

\section{B}

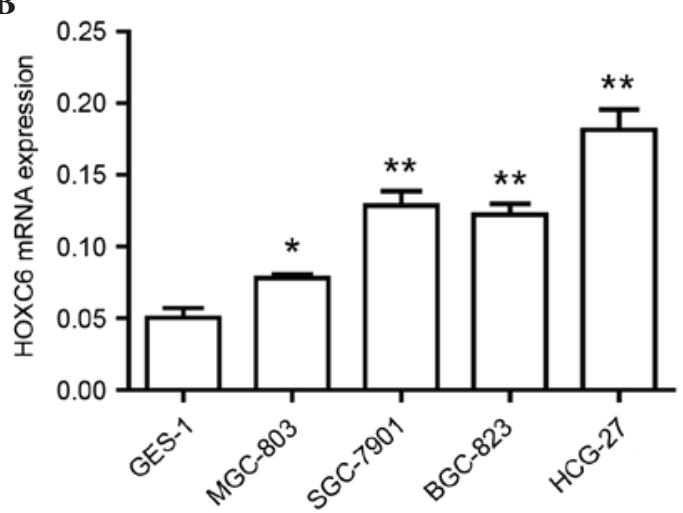

Figure 1. HOXC6 is upregulated in gastric cancer cell lines. (A) Expression profile analysis of HOXC6 using RT-qPCR. (B) The relative mRNA expression levels of HOXC6 were evaluated in gastric cancer cell lines and the GES-1 normal gastric epithelial cell line using RT-qPCR. $\beta$-actin was used as an internal control. ${ }^{*} \mathrm{P}<0.05,{ }^{* *} \mathrm{P}<0.01$ vs. GES-1. RT-qPCR, reverse transcription-quantitative polymerase chain reaction;HOXC6, homeobox C6.

this hypothesis, a Transwell assay with non-pre-coated and pre-coated matrigel was used to determine the migration and invasion abilities of SGC-7901 and BGC-823 gastric cancer cell lines. In the migration experiment, compared with the gastric cancer cells transfected with shRNA-NC, there was a significant decrease in the number of HOXC6 RNAi gastric cancer cells migrating through the Transwell $(\mathrm{P}<0.05$ and $\mathrm{P}<0.01$; Fig. 5). In the invasion assay, it was demonstrated that compared with the gastric cancer cells transfected with shRNA-NC, there were significantly fewer gastric cancer cells with downregulated HOXC6 that moved through the Transwell following degrading the pre-coated matrigel $(\mathrm{P}<0.05$ and $\mathrm{P}<0.01$; Fig. 5). These data demonstrate that HOXC6 promotes migration and invasion of gastric cancer cells.

HOXC6 positively regulated the gene expression of MMP9 by activating ERK signaling in gastric cancer cells. Previous studies have demonstrated the association between HOXC6 expression and tumor invasion depth in gastric cancer tissues $(12,14)$. MMPs are important in the invasion and metastasis of tumor cells. In order to investigate the role of HOXC6 in the mechanism underlying the in vitro invasive and metastatic abilities of gastric cancer cells, RT-PCR was used to analyze the effect of HOXC6 on the expression of MMP2 and MMP9 in SGC-7901 and BGC-823 gastric cancer cells. The results demonstrate that the overexpression of HOXC6 in gastric cancer cells did not affect the mRNA expression levels of MMP2, but significantly upregulated the mRNA expression of MMP9. Following the downregulation of HOXC6 in the gastric cancer cells by transfection with shRNAs, there was a significant downregulation in the expression of MMP9
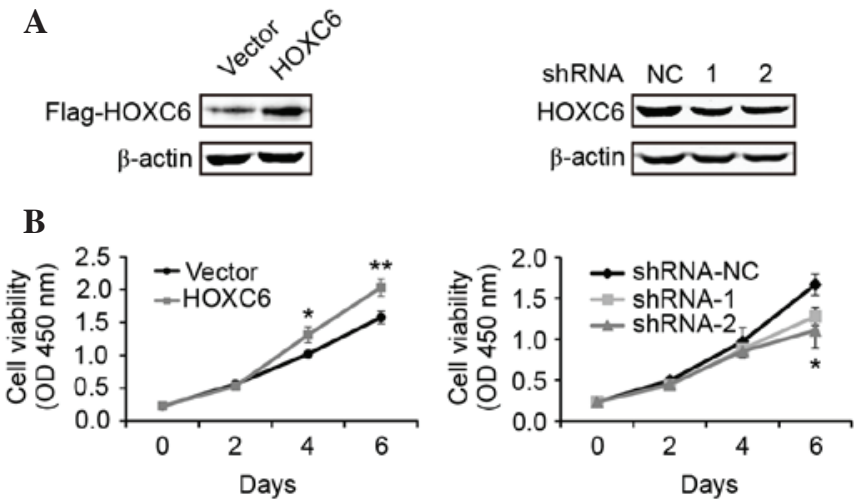

Figure 2. Overexpression and knockdown of HOXC6 regulates cellular proliferation of the GES-1 normal gastric epithelial cell line in vitro. (A) The ectopic HOXC6 protein was detected by western blotting in the GES-1 cell line. (B) HOXC6 affected the proliferation of GES-1 cell line. The data are presented as the mean \pm standard deviation. All the above experiments were repeated three times to ensure the reproducibility of the results. ${ }^{*} \mathrm{P}<0.05$, ${ }^{* *} \mathrm{P}<0.01$ vs. the control. HOXC6, homeobox C6; NC, negative control; OD, optical density; shRNA, short hairpin RNA.

$(\mathrm{P}<0.001)$, however there was no significant change in MMP2 expression (Fig. 6A and 6B).

ERK is the key molecule in regulating expression of MMPs. Thus, the present study detected the activation of ERK in SGC-7901 cells using immunoblotting. Notably, forced HOXC6 expression upregulated the phosphorylation level of ERK, compared with the empty vector control. However, the reverse results were observed with the downregulation of HOXC6 in SGC-7901 cells (Fig. 6C). These results suggest that HOXC6 promoted the in vitro invasive and metastatic abilities of gastric cancer cells, potentially by upregulating the expression of MMP9 via increasing ERK signaling.

\section{Discussion}

The abnormal expression of genes in tumor tissues is often associated with the occurrence and development of tumors $(15,16)$, with the abnormal expression of certain genes being the probable initiating factor of tumorigenesis, while other genes are abnormally expressed as a result of tumorigenesis $(17,18)$. The highly expressed tumor-associated genes may be useful in tumor-targeting treatment aiming to alter their expression or inhibiting associated gene function $(19,20)$. We have reported in a previous study that the HOXC6 gene was highly expressed in gastric cancer clinical tissue samples (12). The present study further investigated the HOXC6 gene expression levels in gastric cancer cell lines. The results indicate that compared with the GES-1 normal gastric mucous epithelial cell line, the level of expression of HOXC6 in a number of common gastric adenocarcinoma cell lines was significantly increased, suggesting that the HOXC6 gene is a highly-expressed tumor-associated gene.

The proliferation of tumor cells is the predominant cause of tumor oppression, obstruction and stenosis of visceral organs $(21,22)$. Interfering with tumor cell proliferation is an important therapeutic strategy for treating tumors. In the present study, gene overexpression and RNAi experiments were conducted and it was demonstrated that the gastric 
A

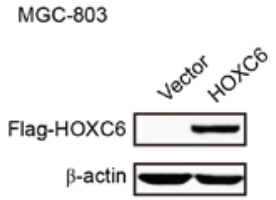

SGC-7901

BGC-823

HCG-27
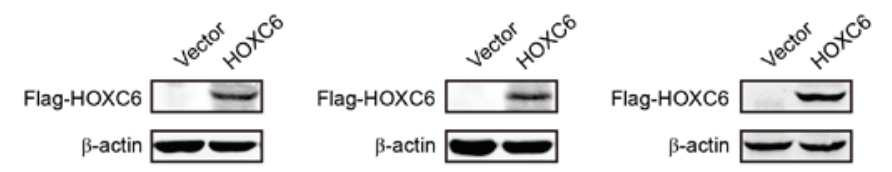

B
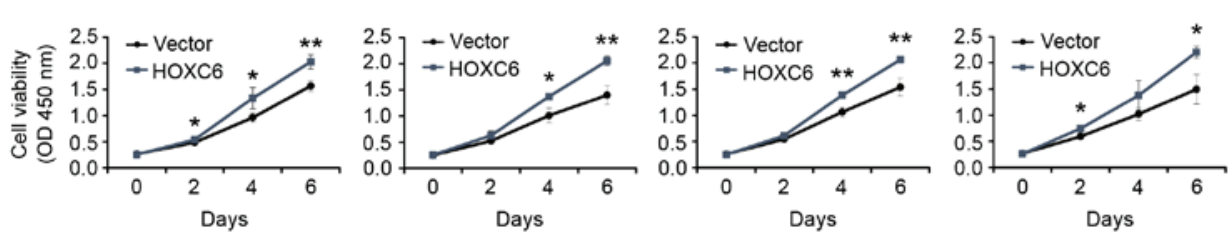

C
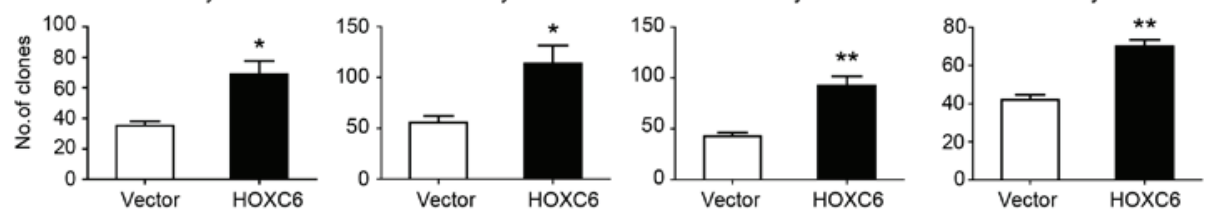

D
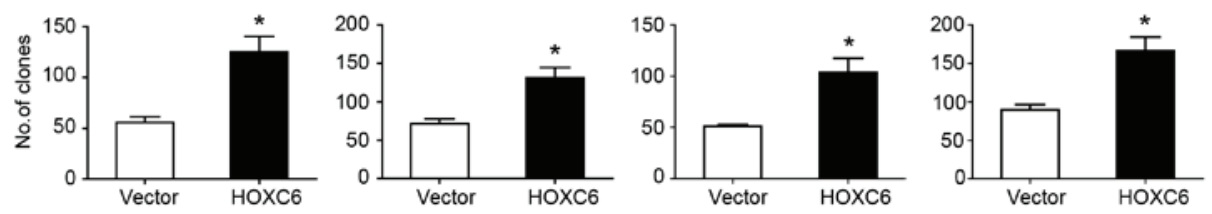

Figure 3. Overexpression of HOXC6 promotes gastric cancer cellular proliferation, colony formation, and soft agar clone formation in vitro. (A) The ectopic HOXC6 protein was detected by western blotting in four gastric cancer cell lines. (B) Ectopic HOXC6 promoted the proliferation of four gastric cancer cell lines. (C) Overexpressed HOXC6 enhanced the colony formation of the gastric cancer cell lines, as demonstrated by representative plates of cells transfected with flag-HOXC6 and empty control vector. (D) Forced HOXC6 expression promoted clone formation in soft agar. The histograms represent the numbers of clones, and the data are presented as the mean \pm standard deviation. All the above experiments were repeated 3 times to ensure the reproducibility of the results. ${ }^{*} \mathrm{P}<0.05,{ }^{* * *} \mathrm{P}<0.01$ vs. the control. HOXC6, homeobox C6; OD, optical density.

A

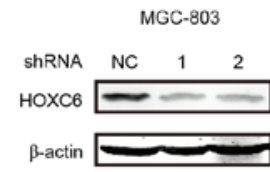

B

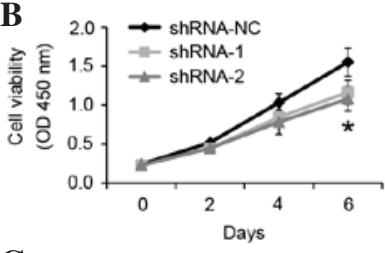

C

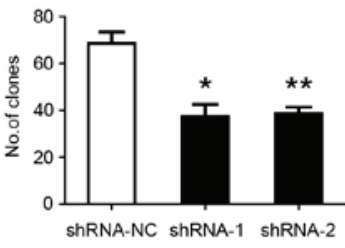

D

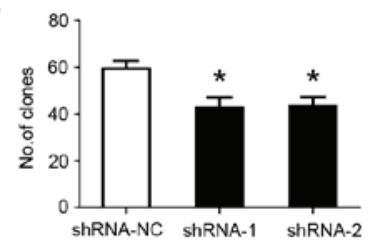

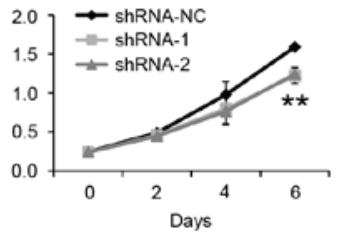
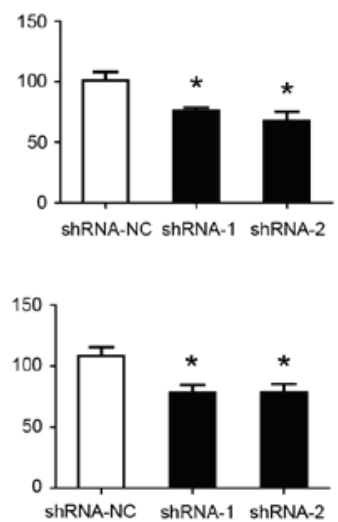

BGC-823

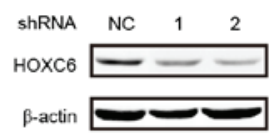

SSRNA NC 112

HOXC6 —- -

-actin $=$
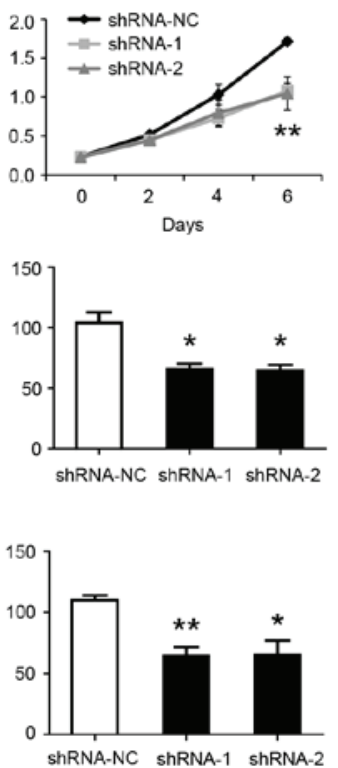
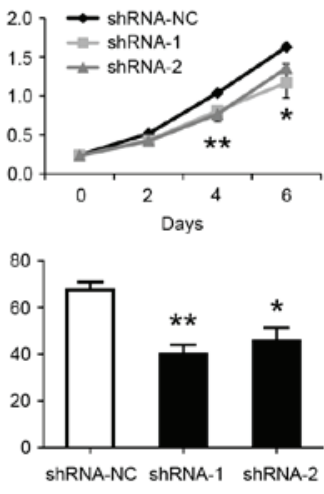

HCG-27

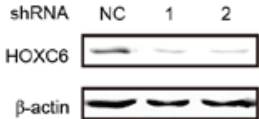

$\beta$-actin

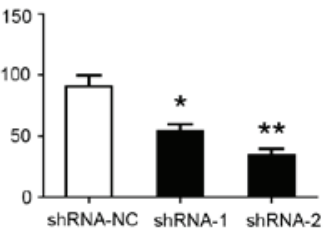

Figure 4. Knockdown of HOXC6 expression inhibits gastric cancer cellular proliferation, colony formation, and soft agar clone formation in vitro. (A) Two constructed shRNAs knocked down endogenous HOXC6 in four gastric cancer cell lines, as determined by an immunoblotting assay. (B) HOXC6 knockdown suppressed the proliferation of four gastric cancer cell lines in vitro. (C) HOXC6 RNAi limited colony formation in the gastric cancer cell lines in vitro, as demonstrated by representative plates of cells transfected with the HOXC6 shRNA and shRNA-NC control. (D) HOXC6 RNAi suppressed colony formation in soft agar. The histograms represent the number of clones, and the data are presented as the mean \pm standard deviation. All the above experiments were repeated 3 times to confirm the reproducibility of the results. ${ }^{*} \mathrm{P}<0.05,{ }^{* *} \mathrm{P}<0.01$ vs. the control. HOXC6, homeobox C6; shRNa, hort hairpin RNA; NC, negative control; RNAi, RNA interference; OD, optical density. 
A
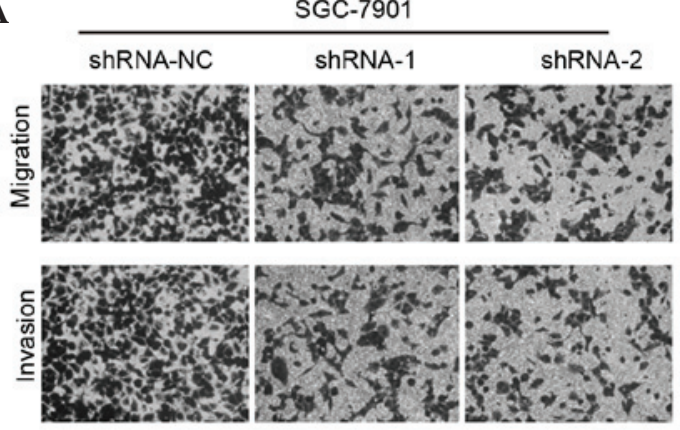

B

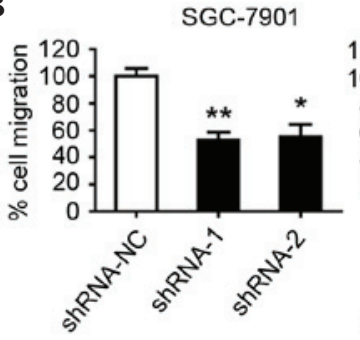

BGC-823

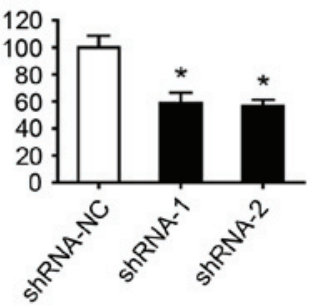

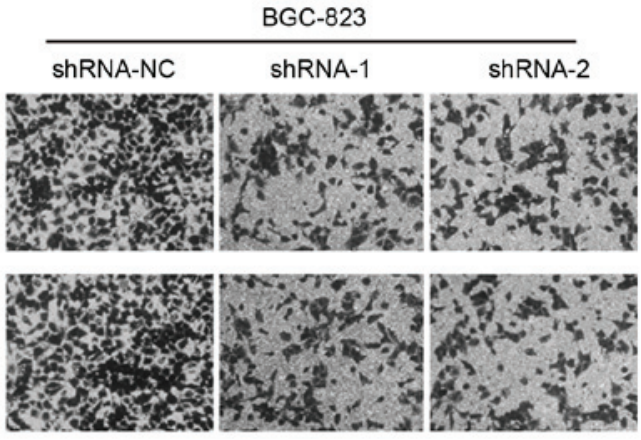

BGC-823

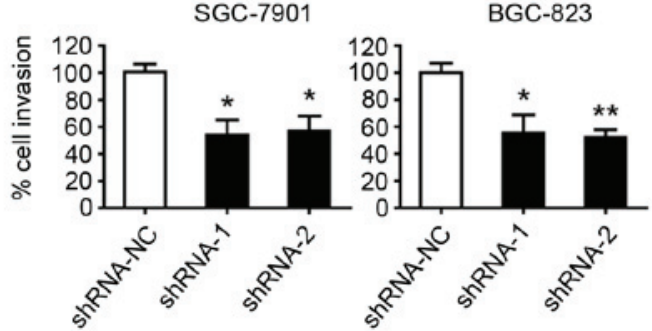

Figure 5. Knockdown of HOXC6 inhibits gastric cancer cellular migration and invasion in vitro. (A) Cell migration and invasion of SGC-7901 and BGC-823 cells transfected with shRNAs were evaluated with Transwell assays. (B) Transwell assays were performed to test the migratory and invasive ability of SGC-7901 and BGC-823 cells. The histograms represent the relative percentage of the gastric cancer cells penetrating the Transwell plates, and the data are presented as the mean \pm standard deviation. All the above experiments were repeated 3 times to confirm the reproducibility of the results. ${ }^{*} \mathrm{P}<0.05$, ${ }^{* *} \mathrm{P}<0.01$ vs. the control. HOXC6, homeobox C6; shRNA, short hairpin RNA.

A

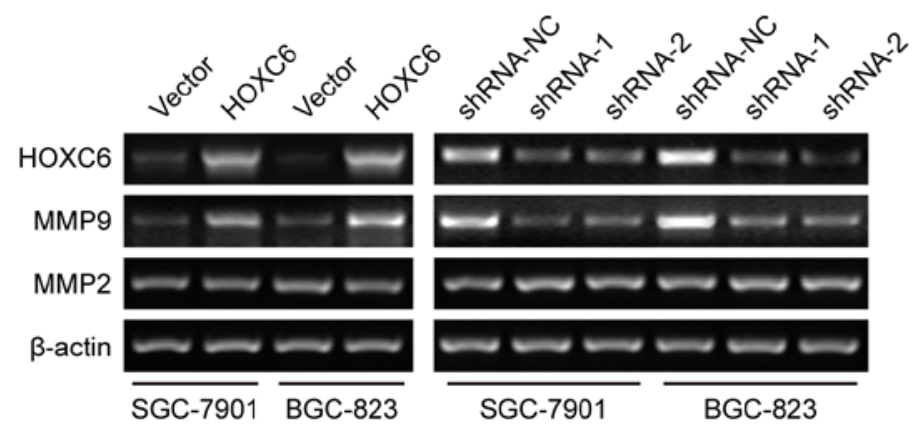

B
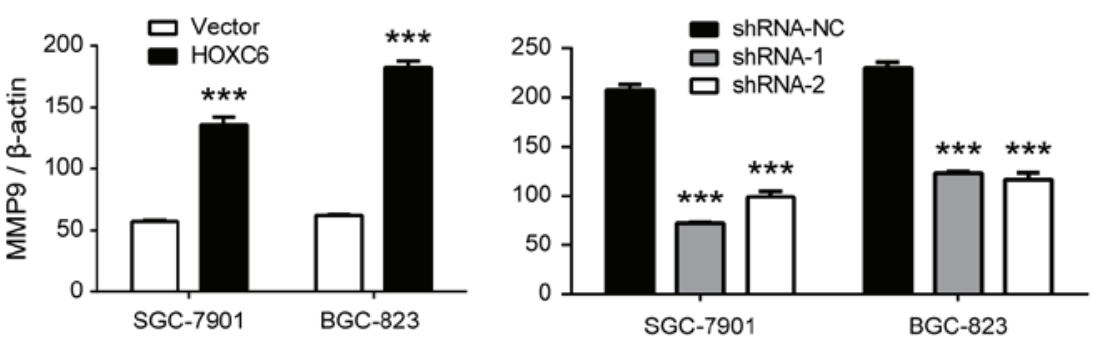

C

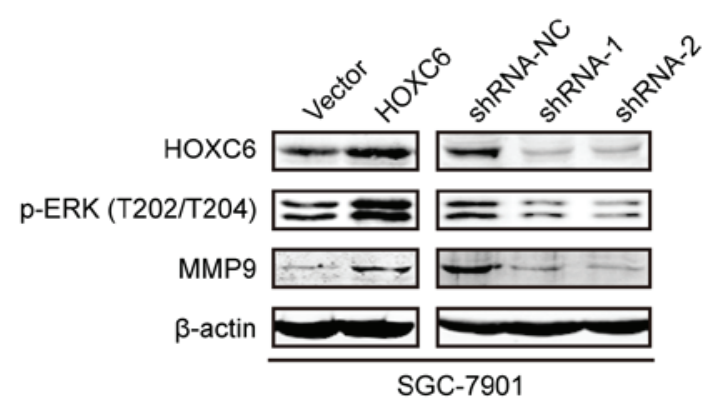

Figure 6. HOXC6 promotes expression of MMP9 by enhancing ERK activation. (A) Expression of MMP9 and MMP2 genes were detected following upregulation and downregulation of HOXC6 by reverse transcription-quantitative polymerase chain reaction in SGC-7901 and BGC-823 gastric cancer cell lines (B) Quantification of the bands was performed by optical density scanning of the results in (A). (C) Phosphorylation levels of ERK and expression of MMP9 were evaluated following upregulation and downregulation of HOXC6 through immunoblotting in SGC-7901 gastric cancer cells. $\beta$-actin served as the internal control. ${ }^{* * *} \mathrm{P}<0.001$ vs. vector or shRNA-NC. HOXC6, homeobox C6; MMP, matrix metalloproteinase; ERK, extracellular-regulated kinase; shRNA, short hairpin RNA. 
cancer-associated HOXC6 gene gained and lost its function, respectively. The results of the experiment indicated that the in vitro overexpression of HOXC6 promoted the proliferation of gastric cancer cells while the downregulation of HOXC6 inhibited the growth of gastric cancer cells, suggesting HOXC6 has similar characteristics as oncogenes in the promotion of cell proliferation.

The predominant cause of poor prognosis of malignant tumors is metastasis $(23,24)$. Previous studies have suggested that HOXC6 was closely associated with poor tumor prognosis, including overexpression of HOXC6 demonstrated to inhibit the differentiation of bone marrow and promote its malignant transformation (25), and HOXC6 expression has been associated with poor prognosis of patients with head and neck tumors (26), nasopharyngeal carcinoma (27), esophageal cancer (5) and gastric cancer (12). A correlation analysis of gastric cancer clinical samples and the clinical pathological features of the disease demonstrated that HOXC6 was also associated with tumor infiltration depth (12). Transwell invasion experiments were used to investigate the effect of HOXC6 gene expression on the invasion and metastasis of gastric cancer cells. The results demonstrated that the HOXC6 gene effectively promoted the invasion and metastasis of gastric cancer cells.

Tumor invasion and metastasis is a multiple step process, tumor cells first infiltrate into the extracellular matrix, then activate associated cells, in addition to synthesizing and secreting associated degradative enzymes in order to destruct the basement membrane and extracellular matrix $(28,29)$. The common enzymes identified during this process are members of the MMP family (30-33). In order to investigate the effect of HOXC6 on the MMP family gene expression, the effect of altering the level of HOXC6 expression on the mRNA expression levels of MMP2 and MMP9 was observed. The results indicated that HOXC6 positively regulated MMP9 expression with no marked effect on the expression level of MMP2. The changes in MMP9 expression levels were regulated by increasing ERK signaling.

In conclusion, the present study demonstrated that HOXC6 is highly expressed in gastric cancer cell lines and that it promoted the proliferation, migration and invasion of gastric cancer cells in vitro. A possible mechanism underlying the invasion of the basement membrane may be upregulation of MMP9 via ERK activation. The current study provides further elucidation of the role of HOXC6 in gastric cancer, which may be useful in the design and evolution of gastric cancer therapeutic strategies.

\section{Acknowledgements}

The present study was supported by grants from the Health Bureau of Shanghai (grant no. 20124453), the Science and Technology Commission of Shanghai Municipality, Pudong New Area (grant no. PKJ2013-Y02) and the Key Speciality Construction Project of Pudong Health and Family Commission of Shanghai (grant no. PWZz2013-06).

\section{References}

1. Shin HR, Carlos MC and Varghese C: Cancer control in the Asia Pacific region: Current status and concerns. Jpn J Clin Oncol 42: $867-881,2012$.
2. Wang J, Song YX and Wang ZN: Non-coding RNAs in gastric cancer. Gene 560: 1-8, 2015.

3. Murayama Y, Oritani K and Tsutsui S: Novel CD9-targeted therapies in gastric cancer. World J Gastroenterol 21: 3206-3213, 2015.

4. Smalley SR, Benedetti JK, Haller DG, Hundahl SA, Estes NC, Ajani JA, Gunderson LL, Goldman B, Martenson JA, Jessup JM, et al: Updated analysis of SWOG-directed intergroup study 0116: A phase III trial of adjuvant radiochemotherapy versus observation after curative gastric cancer resection. J Clin Oncol 30: 2327-2333, 2012.

5. Du YB, Dong B, Shen LY, Yan WP, Dai L, Xiong HC, Liang Z, Kang XZ, Qin B and Chen KN: The survival predictive significance of HOXC6 and HOXC8 in esophageal squamous cell carcinoma. J Surg Res 188: 442-450, 2014.

6. Grier DG, Thompson A, Kwasniewska A, McGonigle GJ, Halliday HL and Lappin TR: The pathophysiology of HOX genes and their role in cancer. J Pathol 205: 154-171, 2005.

7. Chariot A and Castronovo V: Detection of HOXA1 expression in human breast cancer. Biochem Biophys Res Commun 222: 292-297, 1996.

8. Devita G, Barba P, Odartchenko N, Givel JC, Freschi G, Bucciarelli G, Magli MC, Boncinelli E and Cillo C: Expression of homeobox-containing genes in primary and metastatic colorectal-cancer. Eur J Cancer 29A: 887-893, 1993.

9. Fujiki K, Duerr EM, Kikuchi H, Ng A, Xavier RJ, Mizukami Y, Imamura T, Kulke MH and Chung DC: Hoxc6 is overexpressed in gastrointestinal carcinoids and interacts with JunD to regulate tumor growth. Gastroenterology 135: 907-916, 2008

10. Li B, Jin H, Yu Y, Gu C, Zhou X, Zhao N and Feng Y: HOXA10 is overexpressed in human ovarian clear cell adenocarcinoma and correlates with poor survival. Int J Gynecol Cancer 19: $1347-1352,2009$

11. Chen KN, Gu ZD, Ke Y, Li AY, Shi XT and Xu GW: Expression of $11 \mathrm{HOX}$ genes is deregulated in esophageal squamous cell carcinoma. Clin Cancer Res 11: 1044-1049, 2005.

12. Zhang Q, Jin XS, Yang ZY, Wei M, Liu BY and Gu QL: Upregulated Hoxc6 expression is associated with poor survival in gastric cancer patients. Neoplasma 60: 439-445, 2013.

13. Yahagi N, Kosaki R, Ito T, Shimada H, Tomita M, Takahashi T and Kosaki K: Position-specific expression of HOX genes along adult gastrointestinal tract in humans. Congenit Anom (Kyoto) 44: 18-26, 2004.

14. Chen SJ, Lin JP and Si JM: Experimental study on HOXC6 interference suppression malignant biological behavior in gastric cancer. In: Proceedings of the 7th Zhejiang Digestive Disease Academic Congress and the American Gastroenterological Association the First China Academic Forum. China Academic Journal Electronic Publishing House, China, pp88-89, 2014.

15. Hanahan D and Weinberg RA: Hallmarks of cancer: The next generation. Cell 144: 646-674, 2011.

16. Wang YP, Huang LY, Sun WM, Zhang ZZ, Fang JZ, Wei BF, Wu BH and Han ZG: Insulin receptor tyrosine kinase substrate activates EGFR/ERK signalling pathway and promotes cell proliferation of hepatocellular carcinoma. Cancer Lett 337: 96-106, 2013.

17. Abbott KL, Nyre ET, Abrahante J, Ho YY, Isaksson Vogel R and Starr TK: The candidate cancer gene database: A database of cancer driver genes from forward genetic screens in mice. Nucleic Acids Res 43 (Database Issue): D844-D848, 2015.

18. Nadauld LD, Garcia S, Natsoulis G, Bell JM, Miotke L, Hopmans ES, Xu H, Pai RK, Palm C, Regan JF, et al: Metastatic tumor evolution and organoid modeling implicate TGFBR2 as a cancer driver in diffuse gastric cancer. Genome Biol 15: 428, 2014.

19. Barthélémy P, Aftimos P and Awada A: New generations of targeted therapies fighting the resistance in solid tumors. Curr Opin Oncol 27: 243-249, 2015.

20. Rolfo C, Bronte G, Sortino G, Papadimitriou K, Passiglia F, Fiorentino E, Marogy G, Russo A and Peeters M: The role of targeted therapy for gastrointestinal tumors. Expert Rev Gastroenterol Hepatol 8: 875-885, 2014.

21. Cargnello M, Tcherkezian J and Roux PP: The expanding role of mTOR in cancer cell growth and proliferation. Mutagenesis 30 : 169-176, 2015.

22. Yang H, Zhai YY, Hu TT and Han ZG: Expression of PAGE5 and effect on proliferation and migration in hepatocellular carcinoma. Journal of Lanzhou University (Medical Sciences) 41: 23-28, 2015 (In Chinese).

23. Condamine T, Ramachandran I, Youn JI and Gabrilovich DI: Regulation of tumor metastasis by myeloid-derived suppressor cells. Annu Rev Med 66: 97-110, 2015. 
24. Sayegh ET, Burch EA, Henderson GA, Oh T, Bloch O and Parsa AT: Tumor-to-tumor metastasis: Breast carcinoma to meningioma. J Clin Neurosci 22: 268-274, 2015.

25. Wurm M, Kowalski J, Heckl D, Zhang XB, Nelson V, Beard BC and Kiem HP: Ectopic expression of HOXC6 blocks myeloid differentiation and predisposes to malignant transformation. Exp Hematol 42: 114-125.e4, 2014.

26. Moon SM, Kim SA, Yoon JH and Ahn SG: HOXC6 Is deregulated in human head and neck squamous cell carcinoma and modulates Bcl-2 expression. J Biol Chem 287: 35678-35688, 2012.

27. Win KT and Li CF: HOXC6 overexpression as a poor prognostic factor in patients with nasopharyngeal carcinoma. Modern Pathology 27: 331A, 2014 (In Chinese).

28. Subramaniam A, Shanmugam MK, Perumal E, Li F, Nachiyappan A, Dai X, Swamy SN, Ahn KS, Kumar AP, Tan BK, et al: Potential role of signal transducer and activator of transcription (STAT)3 signaling pathway in inflammation, survival, proliferation and invasion of hepatocellular carcinoma. Biochim Biophys Acta 1835: 46-60, 2013.
29. Wells A, Grahovac J, Wheeler S, Ma B and Lauffenburger D: Targeting tumor cell motility as a strategy against invasion and metastasis. Trends Pharmacol Sci 34: 283-289, 2013.

30. Kim HC, Kim YS, Oh HW, Kim K, Oh SS, Kim JT, Kim BY, Lee SJ, Choe YK, Kim DH, et al: Collagen triple helix repeat containing 1 (CTHRC1) acts via ERK-dependent induction of MMP9 to promote invasion of colorectal cancer cells. Oncotarget 5: 519-529, 2014

31. Merdad A, Karim S, Schulten HJ, Dallol A, Buhmeida A, Al-Thubaity F, Gari MA, Chaudhary AG, Abuzenadah AM and Al-Qahtani MH: Expression of matrix metalloproteinases (MMPs) in primary human breast cancer: MMP-9 as a potential biomarker for cancer invasion and metastasis. Anticancer Res 34: 1355-1366, 2014

32. Yang N, Hui L, Wang Y, Yang H and Jiang X: SOX2 promotes the migration and invasion of laryngeal cancer cells by induction of MMP-2 via the PI3K/Akt/mTOR pathway. Oncol Rep 31: 2651-2659, 2014.

33. Zhang W, Liu Y and Wang CW: S100A4 promotes squamous cell laryngeal cancer Hep-2 cell invasion via NF-kB/MMP-9 signal. Eur Rev Med Pharmacol Sci 18: 1361-1367, 2014. 